\title{
BEHAVIOURAL VARIATION IN NATURAL POPULATIONS. IV. MENDELIAN MODELS AND HERITABILITY OF A FEEDING RESPONSE IN THE GARTER SNAKE, THAMNOPHIS ELEGANS
}

FRANK A. AYRES AND STEVAN J. ARNOLD*

University of Oxford, Department of Biomathematics, Pusey Street, Oxford OX1 2JZ, U.K.

Received 14.iii.83

\section{SUMMARY}

Heritability of a feeding response was very similar in two conspecific populations despite divergence in average behaviour. These results support the view that genetic variances evolve more slowly than phenotypic means. One locus models with absolute penetrance gave poor fits to family data from both populations.

\section{INTRODUCTION}

There are excellent prospects for applying the theory of quantitative genetics to the evolution of natural populations. Field workers are increasingly successful at estimating heritabilities and genetic correlations. Recent success in field measurement of fitness indicates that natural selection on metric traits will be routinely measured in the near future. However, one of the obstacles to more general application of theory is the question of evolutionary stability of genetic parameters.

Quantitative genetic equations could be used to model phenotypic evolution if genetic variances and covariances remain relatively constant (Lande, 1976a 1979). Constancy could be expected on theoretical grounds since the loss in genetic variance in each generation due to selection may be balanced by input from migration, polygenic mutation and recombination so that an equilibrium in variation may be achieved (Lande, 1976b; Bulmer, 1980). Selection experiments show that genetic variation may be retained even after many generations of intense directional selection (Falconer, 1981). Thus theoretical and experimental results suggest that genetic architectures might be stable in nature. However, it is difficult to weigh the opposing natural forces that buffet genetic variation and so evolutionary stability of genetic variance is still an unresolved empirical problem. No-one expects genetic variances and covariances to remain unchanged for millennia, but they might evolve so slowly that constancy can be assumed in models for the differentiation of local populations, geographic races and sister species. The problem is considered in this paper by comparing the heritabilities of a behavioural trait in two widely separated populations of the garter snake, Thamnophis elegans, representing two geographic races.

* Permanent address: Department of Biology, University of Chicago, 1103 E. 57th Street, Chicago, IL 60637, U.S.A. 
A geographic comparison of heritability in this garter snake is particularly interesting because the trait in question is of great ecological significance and has recently been subjected to directional selection in nature. California populations of T.elegans are polymorphic for slug-eating tendency with a slug-eating morph predominating in populations that are sympatric with slugs and a slug-refusing morph predominating elsewhere (Arnold, 1977, 1981 a, 1981b). Sympatric populations feed almost exclusively on slugs but allopatric populations eat mostly vertebrate prey (Arnold, $1981 a$; Kephart and Arnold, 1982; Kephart, 1982). Differences in slugeating tendency are congenital, ontogenetically stable and mediated by heritable differences in chemoreceptive response to slug odour (Arnold, 1981a). A genetic basis for the slug-feeding polymorphism has been confirmed by crosses between populations but so far only first generation hybrids have been analysed (Arnold, 1981c). Geographic comparison of chemoreceptive responses to a variety of prey revealed very similar genetic variance-covariance structures but sample sizes were small (Arnold, $1981 b)$.

The aim of this paper is to compare heritability of slug-feeding in a north coastal and an inland population of $T$. elegans from California. The north coastal population is sympatric with slugs, while the inland population is allopatric to these prey. These populations have diverged in phenotypic mean and we wish to determine whether their genetic variances have also evolved. Secondly, since the evolution of a genetic variance may be affected by the number of segregating loci underlying trait variation (Bulmer, 1980), we fit simple Mendelian models to data from both populations. Since we fit only full sib data from a single generation we cannot conduct a strong test for polygenic inheritance but we are able to reject some simple one locus models.

The assumption that litter mates are full sibs is critical to our analyses but well supported by recent work. Female garter snakes mate only once each reproductive season. Insemination induces female nonreceptivity and, in addition, the male transfers a pheromone laden sperm plug that makes the female unattractive to other males (Devine, 1975; Ross and Crews, 1977, 1978). Furthermore insemination immediately causes catabolism of any sperm stored from earlier matings (Halpert et al., 1983).

\section{MAterials AND MEthods}

\section{(i) Subjects and behavioural tests}

The snakes used in behavioural tests were the laboratory born progency of 124 gravid females that were captured at two study populations in northern California, separated by $285 \mathrm{~km}$. Fifty-six females from the north coastal population, Humboldt county, gave birth to 463 progency (mean litter size $=8 \cdot 27 \pm 1 \cdot 02$ s.d., range $=2-18$ ) and 68 females from the inland population, Lassen county, gave birth to 681 progency (mean litter size $=$ $10 \cdot 01 \pm 1.01$ s.d., range $=1-23$ ) under uniform laboratory conditions. Littermates were separated from their mother within $18 \mathrm{~h}$ of birth and were housed in separate, individual cages.

Behavioural tests consisted of slug-eating trials conducted with naive, newborn snakes. Beginning at the age of 14 or 16 days, each snake was 
offered a small, standard sized piece of slug (Ariolimax californicus or $A$. columbianus) on ten consecutive days. The total number of slug pieces eaten constituted each snake's slug-feeding score. These feeding trials were the snakes' first exposure to food and no other food was offered until the trial sequence was completed. Additional details of maintenance and testing procedures are given in Arnold (1981a, $b, c)$.

Two scales were used in data analysis: the original, quasicontinuous scale (ranging from 0-10) and a binary scale, in which consistent slugrefusers were assigned a value of zero and consistent slug-eaters a value of one (fig. 1).

\section{(ii) Statistical methods}

\section{(a) Heritability}

Heritability was estimated using two techniques; weighted family analysis of variance and a jackknife procedure. In each population both techniques were used on binary data and on the original quasicontinuous scale.

Heritability was first estimated using Bulmer's (1980, p. 84) procedure in which families are weighted by the reciprocal of the expected family mean, rather than by family size. This procedure has the effect of weighting families in proportion to our confidence in them as estimates of breeding value. Bulmer gives an equation that must be solved iteratively for the appropriate weighted-among-family component of variance. Bulmer gives the equation and discussion as illustration of the analysis of half sib family data but they can be equally well applied to full sib data. We used a Newton-Raphson iteration procedure that used as starting values the components of within- and among-family variance calculated by conventional means with standard correction for unequal group sizes. Heritability was then estimated as twice the intraclass correlation.

Heritability was also estimated with a jackknife procedure (Quenouille 1949, 1956), using Arvesen's (1969) results for interval estimation in unbalanced variance component problems. In using the jackknife, one family at a time was deleted from the complete population sample. As before, heritability was estimated as twice the intraclass correlation. Arvesen's (1969) results were also used to estimate confidence intervals.

\section{(b) Mendelian models}

One locus, two allele models were fitted to the full sibship data from each population. The most general model allowed incomplete dominance and variable penetrance with the following parameters: the conditional probabilities that individuals of genotypes $A A, A a$ and $a a$ will be slugrefusers $\left(p_{1}, p_{2}\right.$ and $\left.p_{3}\right)$ and the frequency of the $A$ allele $(q)$. We assumed random mating in a population at Hardy-Weinberg equilibrium. Two special cases are of particular interest: complete dominance with absolute penetrance $\left(p_{1}=1, p_{2}=p_{3}=0\right.$, or $\left.p_{1}=p_{2}=0, p_{3}=1\right)$ and complete dominance with variable penetrance $\left(p_{1}=p_{2}\right.$ or $\left.p_{2}=p_{3}\right)$.

We derived an expression for the probability of obtaining $x_{i}$ slug-refusers and $n_{i}-x_{i}$ slug-eaters in a full sibship of size $n_{i}$ under these genetic assumptions by enumerating the expected progeny proportions for each of the six 
possible mating types in the population. The likelihood function was then specified as the product of these expressions over all actual sibship sizes and feeding morph compositions in each population.

Two log likelihood functions were derived: one for the general case and one for the special case of complete dominance. Both are quite complicated and given in Ayres (1981).

We estimated genetic parameters for each population using a conventional maximum likelihood approach. General accounts of the method with genetical applications are given by its originator R. A. Fisher (1941) and by Bailey (1961) and Bulmer (1965). The approach involves finding the parameter values that maximize the log likelihood function. We used an iterative method of numerical approximation, rather than explicit solutions, since the model generally had several parameters. Confidence intervals were found by evaluating second derivatives at the maximum likelihood values and taking their matrix inverse in order to obtain the variancecovariance matrix (Bailey, 1961).

Maxima of the log likelihood function were located by exhaustive search of the parameter space for the model with complete dominance, absolute penetrance and the model with complete dominance and variable penetrance. Such search was impractical for the more general genetical model with four parameters. Instead maxima for each population were found by beginning the search at eight different, widely spaced points in the parameter space.

Heritability was estimated using the parameters estimated by maximum likelihood for the model with complete dominance and variable penetrance. Taking $\left(1-\hat{p}_{2}\right)$ as the value of the genotype $a a$ and $\left(1-\hat{p}_{1}\right)$ as the value of the other two genotypes, we estimated the phenotypic, additive genetic and dominance variances using standard one locus expressions (Bulmer, $1980 ;$ Falconer, 1981). We estimated twice the full sib intraclass correlation, $2 \hat{t}$, as the ratio of the sum of the additive genetic variance and half the dominance variance to the total phenotypic variance in order to have heritability estimates comparable to those estimated directly from the full sib data. Finally, we estimated heritability, $\hat{h}^{2}$, as the ratio of additive genetic to phenotypic variance.

\section{RESUlTS}

Feeding scores were bimodally distributed in both populations but with a preponderance of slug-eating snakes in the north coastal population and a preponderance of slug-refusing snakes in the inland population (fig. 1). In light of these strong bimodalities, it is convenient to recognize two feeding morphs: slug-refusers (feeding score $=0-4$ ) and slug-eaters (feeding score $=5-10$ ). This dimorphic classification of feeding morphs will be referred to as the binary scale (slug-refusers $=0$, slug-eaters $=1$ ).

\section{(i) Heritability estimates}

Estimates of heritability obtained using Bulmer's (1980) procedure for weighting family means and a jackknife procedure are reported in table 1. The two estimation procedures gave very similar results on both the binary and quasicontinuous scales. Confidence limits were only obtained 


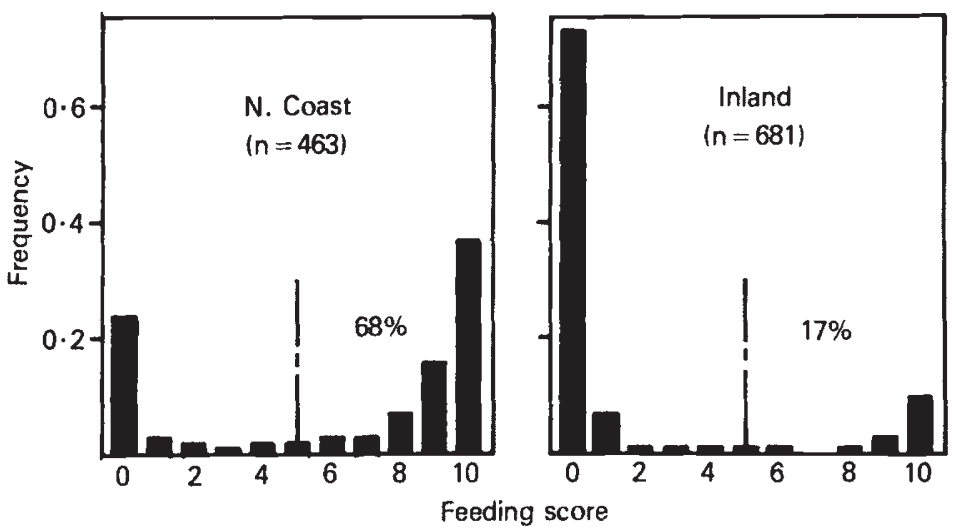

FIG. 1. Frequency distributions of slug-feeding scores (quasicontinuous scale) in two populations of $T$. elegans. The north coastal population is sympatric with slugs but the inland population is allopatric. Percentages indicate the proportions of naive, newborn snakes that were consistent slug-eaters (feeding score $=5-10$ ).

TABLE 1

Heritability estimates for slug-feeding scores in two populations of $\mathrm{T}$, elegans

\begin{tabular}{lll}
\hline \multicolumn{1}{c}{ Estimation Procedure } & North Coastal Population & Inland Population \\
\hline (a) Estimates based on the quasicontinuous scale & \\
Weighted family means & 0.39 & 0.51 \\
Jackknife* & $0.37(0.12-0.62)$ & $0.54(0.23-0.86)$ \\
(b) Estimates based on the binary scale & 0.34 & \\
Weighted family means & $0.31(0.08-0.53)$ & 0.42 \\
Jackknife* & $0.44(0.14-0.73)$ \\
\hline
\end{tabular}

* 95 per cent confidence limits shown in parentheses.

using the jackknife procedure but these clearly indicate statistically significant heterogeneity among families in both populations. Furthermore, the 95 per cent confidence limits for the two populations broadly overlap. There is no evidence for a geographic difference in heritability but quite large samples would be required to detect a true difference as small as $0 \cdot 10$.

\section{(ii) Mendelian models}

One locus models with variable penetrance and complete dominance gave significantly better fits than models with absolute penetrance and complete dominance. Using twice the difference in the values of the log likelihood function at the maximum found for each model as an approximation for chi-square with two degrees of freedom, $\chi_{2}^{2}=20.0 \quad(p<0.01)$ for the north coastal population and $\chi_{2}^{2}=32.7(p<0.01)$ for the inland population.

One locus models that allowed incomplete dominance with variable penetrance gave no better results than models with complete dominance and variable penetrance. A comparison of the best fits in the coastal population gave $\chi_{2}^{2}=0.03$ and in the inland population $\chi_{2}^{2}=0.54$. 
Although there was a single maximum for log likelihood in the simplest model (complete dominance and absolute penetrance), there were local maxima in the other models. For the model with complete dominance and variable penetrance, exhaustive search yielded two local maxima in each of the two populations, barely differing in height within each population (plus a third local maximum in the north coastal population that was much lower than the other two and which will be eliminated from discussion). In one set of solutions for each population, slug-refusal was dominant, the populations showed nearly identical estimates of penetrance but differed greatly in gene frequency (table 2). In the other set of solutions, slug-refusal

\section{TABLE 2}

Maximum likelihood estimates for gene frequency, $\hat{\mathrm{q}}$, and penetrance, $\hat{\mathrm{p}}_{1}$ and $\hat{\mathrm{p}}_{2}$, under an inheritance model with complete dominance. The probabilities that a snake of given genotype will be a slug-refuser are given by $\hat{\mathrm{p}}_{1}$ and $\hat{\mathrm{p}}_{2}$

\begin{tabular}{|c|c|c|c|}
\hline Phenotype & Variable & $\begin{array}{l}\text { North Coastal } \\
\text { Population }\end{array}$ & $\begin{array}{c}\text { Inland } \\
\text { Population }\end{array}$ \\
\hline $\left.\begin{array}{c}A A \\
A a\end{array}\right\} \quad$ slug-refuser & $\hat{p}_{1}$ & $\begin{array}{r}0.9999 \\
( \pm 0 \cdot 1465)\end{array}$ & $\begin{array}{c}0.9344 \\
( \pm 0.0281)\end{array}$ \\
\hline$a a \quad$ slug-eater & $\hat{p}_{2}$ & $\begin{array}{c}0 \cdot 2280 \\
( \pm 0 \cdot 0372)\end{array}$ & $\begin{array}{c}0.2265 \\
( \pm 0 \cdot 1426)\end{array}$ \\
\hline Frequency of $A$ allele, & $\begin{array}{l}\hat{q} \\
\hat{h}^{2} \\
2 \hat{t}\end{array}$ & $\begin{array}{c}0.0752 \\
( \pm 0.0384) \\
0.32 \\
0.32\end{array}$ & $\begin{array}{c}0.6129 \\
( \pm 0.0708) \\
0.25 \\
0.35\end{array}$ \\
\hline
\end{tabular}

( 95 per cent confidence intervals are shown in parentheses).

was recessive, the populations showed very similar penetrance values (nearly the exact reverse of those shown in table 2) and, again, the primary population difference was in gene frequency. Only the parameter estimates corresponding to solutions with slug-refusal dominancy are reported in table 2 since a cross between the two populations suggests this dominance relationship (Arnold, 1981c). Heritability estimates based on this set of solutions are also shown in table 2 and are in good agreement with more direct estimates (table 1).

Three local maxima were found in each population using the model with incomplete dominance and variable penetrance, but none of these gave a better fit than the simpler models (table 2). We cannot eliminate the possibility that undiscovered solutions with the more complicated model would give better fits since the likelihood functions are very complicated and we did not conduct an exhaustive search of the parameter space. The maxima that were found were located in diverse parts of the parameter space. In the coastal population models with overdominance for slug-refusal produced two of the known maxima and a model with dominance for slug-eating produced the third. In the inland population models with dominance for slug-refusal produced two of the known maxima and a model with underdominance for slug-refusal produced the third. 


\section{Discussion}

The present results suggest that heritability of slug-eating behaviour may not have been perturbed during the niche differentiation of $T$. elegans. Estimates were moderately high in both populations with broadly overlapping confidence intervals. In order to conduct a strong test for evolutionary invariance, one would need less ambiguous estimates of additive genetic variance from a whole series of populations. Despite the conspicuous limitations of the data (full sib data from only two populations), the observation of statistically indistinguishable heritabilities is a plausible result. The differentiation of the two populations, though ecologically profound, amounted to a divergence of only 1.5-2 phenotypic standard deviations (Arnold, 1981c). In experimental studies genetic variance can be present after divergence of 10-20 standard deviations and the attainment of a selection limit (Falconer, 1981).

Although the heritability estimates are based on full sib data, they are probably not seriously inflated by common family environment or dominance variance. Common family environments were disrupted the day after birth, maternal diet during gestation has no effect on neonatal prey preferences (Burghardt, 1971) and comparison of reciprocal $F_{1}$ progenies did not reveal any maternal effects on slug-feeding response (Arnold, 1981c). The potential contribution of dominance variance was evaluated with one locus models with complete dominance and found to be small (table 2).

In order to make comparisons of heritability in different taxa it may sometimes be necessary to use results from two or more investigators who have employed different estimation procedures. Consequently it is useful to know how much estimates are affected by differences in estimation procedure. In the present case very similar heritability estimates were obtained when two techniques were applied to two different transformations of scale (table 1). Similar results were obtained when a third estimation procedure was applied to one of these scales, a binary coding of scores. Bulmer (MS) a fitted beta-binomial distribution to the data and estimated the heritabilities to be $0.28 \pm 0.09$ s.e. in the coastal population and $0.33 \pm$ 0.9 s.e. in the inland population. He was able to estimate 95 per cent confidence intervals by a separate technique and these were, respectively $(0 \cdot 13-0.48)$ and $(0 \cdot 15-0.50)$. These results are in close agreement with other estimates and support the observation of geographic invariance in heritability.

Heritability estimates based on tests of chemoreceptive and feeding responses to slugs gave similar results in both populations. Arnold (1981a) estimated the heritability of chemoreceptive response to be 0.17 in both populations. These point estimates are inside the confidence intervals for the feeding score estimates. The somewhat lower chemoreception estimates may reflect larger environmental variance arising from the fact that chemoreception was assayed in just one minute whereas the feeding score averaged behaviour over a ten day period.

The simplest one locus models do not give adequate fits to the slug feeding data from either population. It is evidently necessary to assume that there is incomplete penetrance of geneotypes, but one achieves no additional improvement in fit by assuming incomplete dominance. Thus analysis of full sib data within each population tended to confirm a result 
derived by crossing the two populations. Such crosses indicated simple or directional dominance for slug-refusal (Arnold, 1981c). However, the present results do not resolve the issue of one locus versus polygenic inheritance. One could accommodate incomplete penetrance in the one locus models by invoking additional, modifying loci or by supposing purely environmental effects. Analysis of second generation hybrids could test the polygenic hypothesis and is now in progress.

Acknowledgements. We are grateful to H. Arnold, L. Arnold, D. Kephart and L. Houck for help with field work, to Jean Gladstone for technical assistance, to M. G. Bulmer for much help and advice on data analysis and to M. G. Bulmer, B. Charlesworth and W. G. Hill for helpful discussions. This work was supported by grants from the U.S. National Science Foundation and by a grant from the U.S. Public Health Service.

\section{REFERENCES}

ARNOLD, S. J. 1977. Polymorphism and geographic variation in the feeding behavior of the garter snake Thamnophis elegans. Science, 197, 676-678.

ARNOLD. S. J. 1981 . The microevolution of feeding behavior, In A. Kamil and T. Sargent (eds.), Foraging Behavior: Ecological, Ethological and Psychological Approaches, p. 409453, Garland Press, New York.

ARNOLD, S. J. 1981 b. Behavioral variation in natural populations. I. Phenotypic, genetic and environmental correlations between chemoreceptive responses to prey in the garter snake, Thamnophis elegans. Evolution, 35, 489-509.

ARNOLD, S. J. 1981c. Behavioral variation in natural populations. II. The inheritance of a feeding response in crosses between geographic races of the garter snake, Thamnophis elegans. Evolution, 35, 510-515.

ARVESEN, J. N. 1969. Jackknifing U-statistics. Ann. Math. Statist., 40, 2076-2100.

ARVESEN, J. N. AND SCHMITZ, T. H. 1980. Robust procedures for variance component problems using the jackknife. Biometrics, 26, 677-686.

AYRES, F. A. 1981. A statistical analysis of feeding behaviour in garter snakes. M.Sc. Thesis, Applied Statistics, Oxford.

BAILEY, N. T. J. 1961. Introduction to the Mathematical Theory of Genetic Linkage. Clarendon Press, Oxford.

BUlMER, M. G. 1965. Principles of Statistics. Oliver and Boyd, London.

BUlmer, M. G. 1980. The Mathematical Theory of Quantitative Genetics. Clarendon Press. Oxford.

BULMER, M. G. MS. Estimating the heritability of a binary trait.

BURGHARDT, G. M. 1971. Chemical-cue preferences of newborn snakes: influence of prenatal maternal experience. Science, 171, 921-923.

DEVINE, M. C. 1975. Copulatory plugs in snakes: enforced chastity. Science, 187, 844-845.

FALCONER, D. S. 1981. Introduction to Quantitative Genetics. Longman, London.

FISHER, R. A. 1941. Statistical Methods for Research Workers. Oliver and Boyd, London.

HALPERT, A. P., GARSTKA, W. R. AND CREWS, D. 1983. Sperm transport and storage and its relation to the annual sexual cycle of the female red-sided garter snake, Thamnophis sirtalis parietalis. (MS).

KEPHART, D. G. 1982. Microgeographic variation in the diets of garter snakes. Oecologia, $52,287-291$.

KEPHART, D. G. AND ARNOLD, S. J. 1982. Garter snake diets in a fluctuating environment: a seven-year study. Ecology, 63, 1232-1236.

LANDE, R. 1976a. Natural selection and random genetic drift in phenotypic evolution. Evolution, 30, 314-334.

LANDE, R. $1976 \mathrm{~b}$. The maintenance of genetic variability by mutation in a polygenic character with linked loci. Genet. Res. Camb., 26, 221-235.

LANDE, R. 1979. Quantitative genetic analysis of multivariate evolution, applied to brain: body size allometry. Evolution, 33, 402-416. 
QUENOUILLE, M. H. 1949. Appropriate tests of correlation in timeseries. J. R. Statist. Soc., $B 11,68-84$.

QUENOUILLE, M. H. 1956. Notes on bias in estimation. Biometrika, 43, 353-360.

ROSS, P. AND CREWS, D. 1977. Influence of the seminal plug on mating behaviour in the garter snake. Nature, 267, 344-345.

ROSS, P. AND CREWS, D. 1978. Stimuli influencing mating behavior in the garter snake, Thamnophis radix. Behav. Ecol. Sociobiol., 4, 133-142. 